\title{
ESTUDIO DE LA PERCEPCIÓN DEL CONFORT TÉRMICO, SATISFACCIÓN RESIDENCIAL Y MODELO DE GESTIÓN, DE LOS RESIDENTES DEL ÁREA DE CONSERVACIÓN Y REHABILITACIÓN ENERGÉTICA EN LA CALLE PIRINEOS, SANTA COLOMA DE GRAMENET, ESPAÑA
}

\section{STUDY OF THE PERCEPTION OF THERMAL COMFORT, RESIDENTIAL SATISFACTION AND MANAGEMENT MODEL, OF THE RESIDENTS OF THE CONSERVATION AND ENERGY REHABILITATION AREA IN THE PIRINEOS STREET, SANTA COLOMA DE GRAMENET, SPAIN}

\section{BARÓN RODRÍGUEZ, Alan}

Universidad Politécnica de Cataluña (UPC). Máster en Estudios Avanzados en Arquitectura-Barcelona MBArch, línea de especialización en Gestión y Valoración Urbana y Arquitectónica

Correo electrónico: alanbaron7@gmail.com

\section{ORTIZ RONCALLO, Mariana}

Universidad Politécnica de Cataluña (UPC). Máster en Estudios Avanzados en Arquitectura-Barcelona MBArch, línea de especialización en Gestión y Valoración Urbana y Arquitectónica

Correo electrónico: mortizroncallo@gmail.com

\section{LARREA EGUIGUREN, Alejandra}

Universidad Politécnica de Cataluña (UPC). Máster en Estudios Avanzados en Arquitectura-Barcelona MBArch, línea de especialización en Gestión y Valoración Urbana y Arquitectónica. Doctoranda

Correo electrónico: alejandra larrea@hotmail.com

\section{AMPUDIA FARÍAS, Andrés}

Universidad Politécnica de Cataluña (UPC). Máster en Estudios Avanzados en Arquitectura-Barcelona MBArch, línea de especialización en Gestión y Valoración Urbana y Arquitectónica Correo electrónico: andrespimpo@gmail.com

\section{MARMOLEJO DUARTE, Carlos}

Departamento de Tecnología de la Arquitectura (TA), Centro de Política de Suelo y Valoraciones (CPSV) Universidad Politécnica de Cataluña (UPC)

Profesor Titular de Universidad

Av. Diagonal, 649, Barcelona, Cataluña, 08028, España

Correo electrónico: carlos.marmolejo@upc.edu

Teléfono: +34 934054385

\section{GARCÍA-ALMIRALL, Pilar}

Departamento de Tecnología de la Arquitectura (TA), Centro de Política de Suelo y Valoraciones (CPSV) Universidad Politécnica de Cataluña (UPC)

Catedrática contratada

Av. Diagonal, 649, Barcelona, Cataluña, 08028, España

Correo electrónico: pilar.garcia-almirall@upc.edu

Teléfono: +34 934015873

Palabras Clave: Percepción del usuario, confort térmico, gestión de vivienda, rehabilitación de vivienda; rehabilitación urbana; rehabilitación energética

Key words: User perception; thermal comfort; dwelling management; dwelling rehabilitation; urban rehabilitation; energy rehabilitation

Citación: BARÓN, A. et al. Estudio de la percepción del confort térmico, satisfacción residencial y modelo de gestión, de los residentes del área de conservación y rehabilitación energética en la calle Pirineos, Santa Coloma de Gramenet, España. En: Libro de proceedings, CTV 2018. XII Congreso Internacional Ciudad y Territorio Virtual. "Ciudades y Territorios Inteligentes". UNCuyo, Mendoza, 5-7 septiembre 2018. Barcelona: CPSV, 2018, p. 303-318. 


\section{Resumen}

Para la rehabilitación del parque habitacional en zonas vulnerables, es necesario implementar modelos de gestión innovadores mediante la colaboración público-privada.

El modelo de gestión utilizado en el Área de Conservación y Rehabilitación (ACR) en calle Pirineos, en el municipio de Santa Coloma de Gramenet es pionero en Cataluña ya que no se había logrado una rehabilitación del parque habitacional privado con recursos de los mismos particulares, en el contexto de un área con una población de renta baja. La función del gobierno municipal fue de promotor y mediador con los vecinos, proyectos técnicos y contratistas para la ejecución de la obra. Otro de los factores innovadores es que el Ayuntamiento funge como instancia crediticia, financiando el proyecto y ofreciendo facilidades de pago a los propietarios de las viviendas, por lo que asume el pago inicial a los contratistas, siendo una de las claves para lograr llevar a cabo la rehabilitación. Así, los propietarios tienen facilidades para retornar al Ayuntamiento el coste de las obras, o bien en dos pagos, o bien en pagos fraccionados durante cinco años e incluso inscribiendo la deuda en el registro de la propiedad en función de las condiciones individuales de cada titular.

Considerando lo anterior, la presente investigación pretende conocer con mayor profundidad los cambios percibidos por los usuarios, después de la rehabilitación de sus viviendas, en tres áreas: (I) el confort térmico; (II) la satisfacción residencial; (III) y el modelo de gestión utilizado por el Ayuntamiento de Santa Coloma de Gramenet; para lo cual se realizó una encuesta cerrada, aplicada a las personas mayores de edad, cabezas de familia y que llevan habitando dentro del ACR Pirineos por más de un año, al ser susceptibles de haber notado los posibles cambios producidos por la intervención.

De los resultados encontrados, uno de los más relevantes es la gestión del Ayuntamiento, que en términos generales se percibe como buena y en particular el sistema de pago. En relación con la satisfacción residencial, aún no se percibe ningún cambio trascendental sobre las relaciones sociales entre los vecinos del barrio y edificio, sin embargo, existe una percepción de mejora en la imagen del barrio y en la revalorización de sus viviendas. Respecto al confort térmico interior, la percepción de los usuarios ha mejorado en las viviendas que antes de la rehabilitación eran más frías, sin embargo, no en todos los casos han sentido diferencia en la temperatura de su hogar.

Esta investigación se ha realizado dentro del seminario de la línea de máster en Gestión y Valoración Urbana y Arquitectónica en el marco del proyecto EnerValor (MINECO/FEDER BIA2015-63606-R)

\section{Abstract}

For the rehabilitation of the housing stock in vulnerable areas, it is necessary to implement innovative management models through public-private collaboration.

The management model used in the Conservation and Rehabilitation Area (ACR) on Pirineos Street, in the municipality of Santa Coloma de Gramenet, is a pioneer in Catalonia since it had not achieved a rehabilitation of the private housing stock with resources of the same individuals, in the context of an area with a low income population. The function of the municipal

Citación: BARÓN, A. et al. Estudio de la percepción del confort térmico, satisfacción residencial y modelo de gestión, de los residentes del área de conservación y rehabilitación energética en la calle Pirineos, Santa Coloma de Gramenet, España. En: Libro de proceedings, CTV 2018. XII Congreso Internacional Ciudad y Territorio Virtual. "Ciudades y Territorios Inteligentes". UNCuyo, Mendoza, 5-7 septiembre 2018. Barcelona: CPSV, 2018, p. 303-318. 
government was to promote and mediate with the neighbors, technical projects and contractors for the execution of the work. Another innovative factor is that the City Council acts as a credit institution, financing the project and offering payment facilities to homeowners, so it assumes the initial payment to contractors, being one of the keys to achieving rehabilitation. Thus, the owners have facilities to return to the City Council the cost of the works, either in two payments, or in installments over five years and even inscribing the debt in the property registry depending on the individual conditions of each owner.

Considering the above, the present investigation intends to know in greater depth the changes perceived by users after rehabilitation in three areas: (I) thermal comfort; (II) residential satisfaction; (III) and the management model used by the City Council of Santa Coloma de Gramenet; for which a closed survey was carried out, applied to the elderly, heads of family and who have been living within the Pyrenees ACR for more than a year, being susceptible to having noticed the possible changes produced by the intervention.

Of the results found, one of the most relevant is the management of the City Council, which in general terms is perceived as good and in particular the payment system. In relation to residential satisfaction, there is still no significant change in the social relations between the residents of the neighborhood and the building; however, there is a perception of improvement in the image of the neighborhood and in the revaluation of their homes. With regard to indoor thermal comfort, the perception of users has improved in homes that were colder before the rehabilitation, but not in all cases they have felt difference in the temperature of their home.

This research has been carried out within the seminar of the master's degree in Management and Urban and Architectural Assessment within the framework of the EnerValor project (MINECO/FEDER BIA2015-63606-R)

\section{Introducción}

El proyecto de ejecución de "Rehabilitación de los edificios incluidos en el Área de Conservación y Rehabilitación (ACR) de la calle Pirineos entre Bruc y Olot" ejecutado por el Ayuntamiento de Santa Coloma de Gramenet, dentro de su Programa de actuación municipal "Renovemos los barrios" fue considerado en mayo de 2016 por la Federación de Municipios de Catalunya (FMC) y la Fundación Carles Pi i Sunyer de Estudios Autonómicos y Locales como un modelo de gestión innovador de "Buena Práctica Municipal" en la temática de vivienda. Adicionalmente en julio del mismo año, la Asamblea del Foro de Entidades para la Implementación de la Estrategia Catalana para la Renovación Energética de Edificios, reconoció la excelencia del programa y su potencial como proyecto modélico, por el que el Ayuntamiento recibió el "Diploma Tractor de la Estrategia Catalana de Eficiencia Energética".

El modelo innovador dentro de los esquemas de gestión urbanística, busca la mejora de las condiciones de habitabilidad de los edificios residenciales de más de 30 años de antigüedad y la mejora de las deficiencias de conservación y mantenimiento, mediante la colaboración público-privada entre el Ayuntamiento y las comunidades de propietarios y residentes. En este modelo el gobierno municipal actúa como promotor y mediador con los vecinos, adjudica los proyectos técnicos, vela por el correcto desarrollo de los trabajos, asume el pago inicial y financia el proyecto ofreciendo facilidades de pago a los propietarios, siendo una de las principales claves para superar la reticencia vecinal a realizar las obras y uno de los factores

Citación: BARÓN, A. et al. Estudio de la percepción del confort térmico, satisfacción residencial y modelo de gestión, de los residentes del área de conservación y rehabilitación energética en la calle Pirineos, Santa Coloma de Gramenet, España. En: Libro de proceedings, CTV 2018. XII Congreso Internacional Ciudad y Territorio Virtual. "Ciudades y Territorios Inteligentes". UNCuyo, Mendoza, 5-7 septiembre 2018. Barcelona: CPSV, 2018, p. 303-318. 
innovadores del modelo. El Ayuntamiento declara un área de conservación y rehabilitación (ACR), integra una treintena de edificios para optar a ofertas más competitivas y reducir costos y además asume el costo de los estudios técnicos. La práctica inició con una Prueba Piloto en el ACR de la calle Pirineos. Para Núria Parlón i Gil, Alcaldesa de Santa Coloma de Gramenet, según lo expuesto dentro del Congreso de Vivienda Social en Barcelona Construmat 2017 "la prioridad es la dignificación del barrio, para que los vecinos se vuelvan a sentir orgullosos y se vuelvan a apropiar de él" (Parlón i Gil, 2017).

Teniendo en cuenta lo anterior, el presente artículo pretende dar a conocer con mayor profundidad los cambios en el confort térmico que los usuarios del proyecto pueden percibir con la reforma realizada, así como los cambios en la satisfacción residencial que los vecinos pueden tener frente a su edificio y su barrio con la rehabilitación de fachadas y finalmente su percepción frente al modelo de gestión que, en un contexto de recursos limitados, tanto públicos como privados, ha permitido realizar una de las primeras intervenciones a escala barrial de rehabilitación del parque residencial con recursos privados en España.

Se inicia con una breve descripción del caso de estudio, sus antecedentes, los estudios preliminares realizados, la delimitación del Área de Conservación y Rehabilitación y finalmente el proyecto y su modelo de gestión. Posteriormente, se hace una breve explicación de la metodología empleada para el desarrollo del estudio, y se describen los resultados encontrados luego de la aplicación y análisis de la encuesta. El artículo finaliza con algunas conclusiones adicionales frente a los análisis de tendencia realizados.

\section{Caso estudio - ACR calle Pirineos, Santa Coloma de Gramanet}

\subsection{Delimitación del Área de Conservación y Rehabilitación}

Una vez identificados los problemas técnicos y sociales analizados en diversos estudios realizados entre 2011 al $2014^{1}$, y gracias al amparo de distintos ordenamientos, principalmente de la Ley del Derecho a la Vivienda y de la Ley de Rehabilitación, Regeneración y Renovación Urbanas; el Ayuntamiento decide introducir un cambio completo en la manera de abordar la cuestión de la rehabilitación de las zonas sensibles y propone como instrumento, la delimitación de un área de Conservación y Rehabilitación - ACR (Ayunt. de Santa Coloma de Gramenet, 2014), amparando su actuación en los siguientes preceptos normativos:

a. Ley 18/2007, 28 de diciembre, del Derecho a la Vivienda; Artículo 30², Artículo 36.13, Artículo $37^{4}$ y Artículo $39^{5}$. (Boletín Oficial del Estado, 2017)

\footnotetext{
Actualización de los indicadores de la Ley de Barrios en el Área Metropolitana de Barcelona (AMB) (2011); "Tipología de edificios de viviendas en Santa Coloma de Gramenet" (Barcelona Regional, 2012); "Rehabilitación energética en las islas residenciales de Santa Coloma de Gramenet", ejecutado por el Grupo de Energía Térmica y Edificación del Instituto de Investigación en Energía de Cataluña, IREC en colaboración con el Departamento de Construcciones Arquitectónicas de la Escuela Técnica Superior de Arquitectura de Barcelona de la Universidad Politécnica de Cataluña, y el Ayuntamiento de Santa Coloma de Gramenet (Ayuntamiento de Santa Coloma de Gramenet, 2014).

2 Artículo 30: Señala los deberes de conservación y rehabilitación de los inmuebles.

Artículo 36.1: Concede a las Administraciones la utilización del instrumento con la finalidad de promover la rehabilitación de inmuebles en áreas especialmente degradadas o por evitar procesos que puedan comportar riesgos para la cohesión social.

4 Artículo 37: Indica el alcance de la declaración de un área de conservación y rehabilitación.

5 Artículo 39: Establece las bases para firmar convenios de rehabilitación con los propietarios y/o personas interesadas en el procedimiento, en caso de que se quieran llevar a cabo.

Citación: BARÓN, A. et al. Estudio de la percepción del confort térmico, satisfacción residencial y modelo de gestión, de los residentes del área de conservación y rehabilitación energética en la calle Pirineos, Santa Coloma de Gramenet, España. En: Libro de proceedings, CTV 2018. XII Congreso Internacional Ciudad y Territorio Virtual. "Ciudades y Territorios Inteligentes". UNCuyo, Mendoza, 5-7 septiembre 2018. Barcelona: CPSV, 2018, p. 303-318. 
b. Ley 8/2013, 26 de junio, de Rehabilitación, Regeneración y Renovación Urbanas; Artículos 7, 8 y 96; Artículo 9.27; Artículos 10, 11 y $12^{8}$. (Boletín Oficial del Estado, 2017)

c. Texto Refundido de la Ley del Suelo (RDL 2/2008), 26 de junio, (TRLS): Artículo 99. (Boletín Oficial del Estado, 2017)

d. Texto Refundido de la Ley de Urbanismo (DL 1/2010), 3 de agosto, (TRLU): Artículo 19710. (Boletín Oficial del Estado, 2017)

e. Ley de Ordenación de la Edificación (38/1999), de 5 de noviembre, (LOE): Artículo 3.111 y 312. (Boletín Oficial del Estado, 2017)

En el año 2013 el Ayuntamiento inicia con la delimitación de un ámbito de trabajo en función de las características especialmente delicadas del parque residencial, en torno de la calle Pirineos. Hace un reconocimiento de las fincas, mediante la inspección visual y en función de los datos disponibles de los estudios previos e identifica los edificios susceptibles de actuación, es decir, aquellos que posiblemente sea necesario rehabilitar (Banco de Buenas Prácticas, 2016).

En el 2014 el Ayuntamiento realiza la inspección inicial de los edificios, mediante visitas técnicas para valorar las necesidades de rehabilitación de cada finca y el estudio económico, con una estimación del coste de las obras a realizar en cada edificio, así como la repercusión por vivienda según coeficientes de propiedad (Banco de Buenas Prácticas, 2016).

a. A partir de las conclusiones del análisis y diagnóstico expuesto, se justificó la delimitación de un ÁCR en la calle Pirineos, entre las calles de Bruc y Olot, con base en las siguientes motivaciones (Ayuntamiento de Santa Coloma de Gramenet, 2014):

b. Los edificios tienen una antigüedad igual o superior a 40 años, lo que, combinado con los bajos niveles de exigencia en la construcción de aquellos años, los hace susceptibles de degradación.

c. El perfil de tenencia de las viviendas, con menos del $50 \%$ ocupado por sus propietarios, dificulta la gestión de su conservación y rehabilitación, por la despreocupación de aquellos propietarios no residentes. También se detecta un alto número de viviendas vacías.

d. Se manifiesta un incumplimiento generalizado por parte de los propietarios de los edificios, del deber legal de conservación establecido por la legislación estatal (texto refundido de la Ley del suelo) y por la legislación autonómica (texto refundido de la Ley de Urbanismo y Ley del Derecho a la Vivienda).

e. Se ha comprobado la existencia de situaciones de insuficiencia y degradación de los requisitos básicos de la edificación, sobre todo en cuanto a los requisitos de habitabilidad y en segundo término los requisitos de seguridad y funcionalidad, lo que hace que los

\footnotetext{
6 Artículos 7,8 y 9: Establecen qué tipo de actuación están permitidas por la ley, define los sujetos obligados y la iniciativa en la ordenación de las actuaciones (artículo 7. Objeto de las actuaciones; artículo 8. Sujetos obligados; y artículo 9. Iniciativa en la ordenación de las actuaciones).

Artículo 9.2: Faculta a las administraciones públicas la adopción de medidas que aseguren la realización de las obras de conservación y ejecución de actuaciones de rehabilitación edificatoria, de regeneración y renovación urbanas que sean precisas y, en su caso, que formulen y ejecuten los instrumentos que las establezcan, cuando existan situaciones de insuficiencia o degradación de los requisitos básicos de funcionalidad, seguridad y habitabilidad de las edificaciones; obsolescencia o vulnerabilidad de barrios, de ámbitos, o de conjuntos urbanos homogéneos; o situaciones graves de pobreza energética.

8 Artículo 10,11, 12,13: Establece los procedimientos y reglas a seguir y las formas de ejecución (10. Reglas básicas para la ordenación y ejecución de las actuaciones; 11. Memoria de viabilidad económica; 12. Efectos de la delimitación de los ámbitos de gestión y ejecución de las actuaciones; y 13. Las formas de ejecución).

9 Artículo 9: Contenido del derecho de propiedad del suelo: deberes y cargas.

10 Artículo 197: Deberes legales de uso, conservación y rehabilitación y órdenes de ejecución.

11 Artículo 3.1. Establece cuáles son los requisitos básicos de la edificación que constituyen el deber legal de conservación de las edificaciones a que hace referencia el artículo 9.1 del texto refundido de la ley del suelo.

12 Artículo 3. Establece los requisitos básicos de la edificación.
}

Citación: BARÓN, A. et al. Estudio de la percepción del confort térmico, satisfacción residencial y modelo de gestión, de los residentes del área de conservación y rehabilitación energética en la calle Pirineos, Santa Coloma de Gramenet, España. En: Libro de proceedings, CTV 2018. XII Congreso Internacional Ciudad y Territorio Virtual. "Ciudades y Territorios Inteligentes". UNCuyo, Mendoza, 5-7 septiembre 2018. Barcelona: CPSV, 2018, p. 303-318. 
edificios se encuentren actualmente en un estado de degradación que requiere la realización de obras.

f. Dado que ni los propietarios individualmente ni las comunidades de propietarios tienen predisposición de realizar las obras de rehabilitación, en parte debido a que muchos de ellos no son propietarios residentes (no se interesan por la mejora de los edificios) y en parte a la situación económica de los propietarios residentes (no disponen de recursos económicos para acometer las obras) se hace necesaria la intervención del Ayuntamiento, al amparo de la Ley de Rehabilitación, Regeneración y Renovación Urbanas.

El 25 de junio de 2014 la Junta de Gobierno Local acuerda la aprobación inicial de la Declaración de un ACR en el ámbito de Pirineos, entre las calles Bruc y Olot. Este documento determina la necesidad de rehabilitación en 32 de los 52 edificios que conforman el ámbito de delimitación con un presupuesto de 1'685.352,72 €. El 16 de diciembre de 2014 la Junta de Gobierno Local acuerda la aprobación definitiva del ACR (Ayuntamiento de Santa Coloma de Gramenet, 2014)

\subsection{Modelo de gestión}

Dentro del sistema de gestión propuesto por el Ayuntamiento, se implementaron tres aspectos que han sido de vital importancia para el éxito del proyecto:

a. Comisión de Seguimiento: formada por los presidentes y/o secretarios de las comunidades de propietarios afectadas, por técnicos del Ayuntamiento y por representantes políticos, la función es garantizar los principios de información, participación y transparencia durante todo el proceso de contratación y ejecución de las obras.

b. Control y supervisión municipal: durante la ejecución de los contratos de servicios y obras de rehabilitación, con el fin de asegurar su correcta ejecución y velar por el interés público.

c. Esquema de financiación y los sistemas de pago: cuya innovación en el esquema de gestión permitió la ejecución del proyecto. Consiste en tres modalidades de pago adecuadas a diferentes tipos de propietarios, teniendo en cuenta su situación. La primera modalidad de pago corresponde al 50-50, el $50 \%$ del valor al inicio de las obras y el $50 \%$ al finalizar la obra de del edificio, esta modalidad es aplicada a personas jurídicas o aquellos residentes que lo eligen (o no se han adherido a las otras fórmulas). La segunda modalidad corresponde al pago de cuotas mensuales durante cinco años (60 cuotas), solución aplicada a personas físicas, quienes deben domiciliar el pago. $\mathrm{Y}$ la tercera modalidad aplicada como inscripción en el registro, aplicable para aquellos propietarios residentes en la vivienda, con ingresos anuales inferiores a $20.000 €$.

\section{Metodología}

Teniendo en cuenta que el proyecto al momento de este estudio, aún se encontraba en la etapa de ejecución de obras (fase 5) y dado que uno de los objetivos del presente trabajo consiste en la evaluación del confort térmico, se delimitó el área de estudio a las Fases 1, 2 y 3 que estaban concluidas (¡Error! No se encuentra el origen de la referencia.).

Citación: BARÓN, A. et al. Estudio de la percepción del confort térmico, satisfacción residencial y modelo de gestión, de los residentes del área de conservación y rehabilitación energética en la calle Pirineos, Santa Coloma de Gramenet, España. En: Libro de proceedings, CTV 2018. XII Congreso Internacional Ciudad y Territorio Virtual. "Ciudades y Territorios Inteligentes". UNCuyo, Mendoza, 5-7 septiembre 2018. Barcelona: CPSV, 2018, p. 303-318. 
Figura 1. Fases de Ejecución del Proyecto

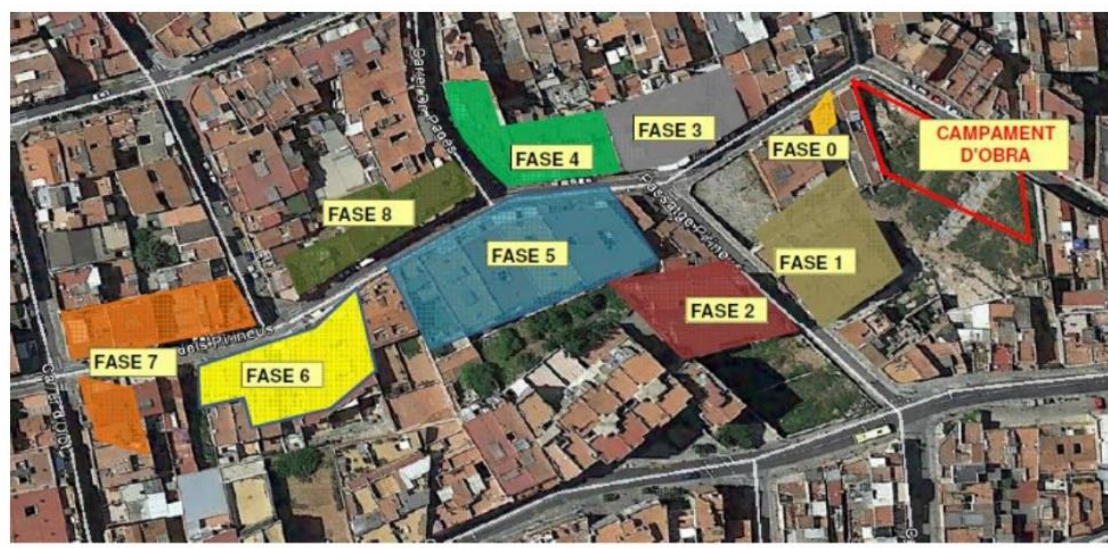

Fuente: Ayuntamiento Santa Coloma de Gramenet

La presente investigación tiene como objetivo conocer con mayor profundidad los cambios percibidos por los usuarios, principalmente en tres aspectos: (I) el confort térmico que los usuarios puedan percibir con la rehabilitación realizada dentro de sus edificios; (II) la percepción entendida como satisfacción residencial que los vecinos puedan tener frente a la mejora física realizada dentro del proyecto y la interacción social a nivel barrial, edificio y vivienda; (III) y la percepción de los residentes frente al modelo de gestión realizado por parte del Ayuntamiento.

Teniendo en cuenta lo anterior, es importante aclarar que al evaluar el confort térmico se pretendía valorar el bienestar o comodidad material de los usuarios frente al buen funcionamiento de las actividades en su residencia en relación con las nuevas condiciones térmicas que podrían proporcionar los cambios de las obras realizadas.

Para esta investigación se utilizó el concepto de satisfacción residencial como un indicador para conocer el impacto social que ha generado el proyecto de rehabilitación de la calle Pirineos. Según lo dicho por Von Breymann (2014) el concepto es un "indicador de bienestar" utilizado desde hace algunas décadas para "medir el agrado del residente con su entorno y/o vivienda" (Von Breymann, 2014). No obstante, García-Almirall (2012) resalta la importancia de situar a la vivienda dentro de un ámbito más amplio, el cual colabora con la identidad del usuario, su calidad de vida y satisfacción. Dicho lo anterior, "la satisfacción "residencial" no puede ser limitada únicamente a la residencia vista como "la vivienda", si no que de implicar a su vez el edificio en general (refiriéndonos a viviendas multifamiliares), el entorno en el que está implantado y las personas con las que convive en su vida cotidiana." (García-Almirall, 2012, pág. 5).

\subsection{Elaboración de la encuesta}

Para realizar el presente estudio fue necesario la implementación de una encuesta cerrada. Dicha encuesta fue aplicada a las personas cabezas de familia, que llevan habitando dentro del ACR Pirineos por más de un año y son mayores de edad.

La encuesta fue dividida en seis secciones: (I) Descripción de la vivienda y el edificio; (II) Hábitos de uso; (III) Confort en su vivienda; (IV) Desarrollo del Proyecto; (V) Valoración residencia; (VI) Datos generales del encuestado.

Citación: BARÓN, A. et al. Estudio de la percepción del confort térmico, satisfacción residencial y modelo de gestión, de los residentes del área de conservación y rehabilitación energética en la calle Pirineos, Santa Coloma de Gramenet, España. En: Libro de proceedings, CTV 2018. XII Congreso Internacional Ciudad y Territorio Virtual. "Ciudades y Territorios Inteligentes". UNCuyo, Mendoza, 5-7 septiembre 2018. Barcelona: CPSV, 2018, p. 303-318. 


\subsection{Mesa de trabajo con el Ayuntamiento}

Se realizó una mesa de trabajo con las personas encargadas del proyecto dentro del Departamento de Urbanismo del Ayuntamiento. El objeto de la sesión fue conocer su opinión y sugerencias sobre la encuesta por aplicar, al ser éstos más conocedores del proyecto y de los vecinos de la actuación

\subsection{Obtención de muestra para aplicación de encuesta}

Para la investigación solo se tomaron en cuenta los edificios que terminaron la rehabilitación antes de noviembre de 2016 (Fases 1, 2 y 3). Esto delimitó el estudio a 8 edificios, para un total de 115 viviendas y 6 locales. Se identificaron viviendas que fueron eliminadas de la muestra porque su fachada no fue afectada por la intervención ( 9 viviendas) y porque estaban en propiedad de bancos o correspondían a viviendas vacías (4), esto daba un universo inicial de 102 posibles encuestas. Con un grado de confianza del 95\%, sería necesario encuestar 80 personas que cumplan con los criterios establecidos.

\subsection{Estrategia de difusión}

Se llevaron a cabo dos estrategias de difusión para dar a conocer los objetivos y medios en que se realizarían las encuestas, en primera instancia se organizó en conjunto con el Ayuntamiento una reunión con los Presidentes de los edificios que eran parte del área de estudio. La segunda estrategia fue el diseño de unos carteles informativos en donde contenía la información básica del estudio, y con la intensión de lograr agendar reuniones con las personas para la aplicación de la encuesta se dejó una especie de calendario, el cual no dio resultado ya que ninguna persona anotó su disponibilidad.

La aplicación de la encuesta se realizó por parte del equipo de investigación de manera presencial del 15 al 28 de mayo de 2017, asistiendo vivienda por vivienda dentro de dos franjas horarias (matutina y vespertina). Se usó diferentes estrategias de proximidad con los vecinos: (I) dejando las encuestas puerta por puerta para que cada vecino pudiera llenarlas y después el equipo de investigación pasaría a recogerlas; (II) haciendo la encuesta personalmente en sus viviendas; (III) acompañando a los vecinos a sus labores diarias, mientras contestaban; (IV) dentro de sus lugares de encuentro (bares, cafés, tienda de proximidad), dichas acciones fueron fundamentales para alcanzar el número de encuestas terminadas.

\subsection{Datos}

El trabajo de campo para la aplicación de la encuesta, presentó un alto grado de complejidad. Del total de las 102 encuestas por realizar, se efectuaron 33 encuestas que equivalen al $32 \%$ del universo inicial, esto corresponde a un error del $11 \%$ con un nivel de confianza del $90 \%$.

En 35 pisos no se encontró a nadie o no abrieron la puerta (34\%), estos corresponden a los casos en los que no se logró contactar personalmente con ningún habitante. El $22 \%$ de los residentes o inquilinos manifestaron que no querían contestar la encuesta, principalmente por factores personales, falta de interés o de tiempo. Asimismo, un $4 \%$ no hablan castellano, por lo tanto, tampoco pudieron contestar la encuesta, en consideración al sesgo de información que esta barrera idiomática podría generar. Finalmente, el $8 \%$ de los casos que no cumplen los requisitos mínimos para diligenciar la encuesta, estos corresponden a personas con tiempo de permanencia en la vivienda inferior a un año o posible ocupación ilegal.

Citación: BARÓN, A. et al. Estudio de la percepción del confort térmico, satisfacción residencial y modelo de gestión, de los residentes del área de conservación y rehabilitación energética en la calle Pirineos, Santa Coloma de Gramenet, España. En: Libro de proceedings, CTV 2018. XII Congreso Internacional Ciudad y Territorio Virtual. "Ciudades y Territorios Inteligentes". UNCuyo, Mendoza, 5-7 septiembre 2018. Barcelona: CPSV, 2018, p. 303-318. 
Dicho lo anterior, los resultados que se presentarán en esta investigación están basados en las tendencias marcadas dentro de las 33 encuestas completadas satisfactoriamente.

\section{Resultados}

\subsection{Perfil de las personas encuestadas}

Del total de los encuestados el 58\% fueron hombres y el 55\% nacieron en España, de los segundos mencionados el $41 \%$ viven en el barrio desde hace más de 10 años, mientras el $22 \%$ de los extranjeros llevan residiendo ahí de 5 a 10 años.

Todas las personas nacidas en el extranjero viven en alquiler, en su mayoría (91\%) se encuentran entre los 25 y 40 años de edad.

De otro lado, el $64 \%$ de las personas encuestadas son propietarios de sus viviendas, de los cuales el $85 \%$ son españoles, y de ellos, el $33 \%$ son mayores de 65 años.

Resalta que el $50 \%$ de los hogares se componen por 4 o más personas, siendo un porcentaje elevado si se considera que el tamaño medio de los hogares en España es de 2.51 personas (INE, 2016).

Nos encontramos que sólo 2 de los encuestados no cuentan con ningún grado de estudios, no obstante, se sitúan en el $44 \%$ de los hogares que tiene un ingreso mensual de 1.000 a 2.000 euros. Asimismo, el $24 \%$ mencionó que el ingreso mensual por hogar es de hasta 500 euros, estando muy por debajo del nivel nacional. Por otro lado, se identificó que los ingresos no tienen una relación directa con el nivel de estudios. Adicional a lo anterior, el $36 \%$ señaló que el ingreso mensual del hogar es insuficiente para solventar los gastos de la vivienda (hipoteca 0 alquiler, consumos, rehabilitación y mantenimiento). Por lo tanto, considerando los ingresos mensuales percibidos por hogar, nos encontramos que el $52 \%$ de los hogares encuestados viven en pobreza energética (identificada en la EPBD como pobreza de combustible), ya que destinan más del $10 \%$ de sus ingresos al pago de las facturas de electricidad y/o gas.

Por último, de los encuestados que se encuentran ocupados, el $50 \%$ trabaja como empleados de comercio o en trabajo no cualificado, siendo el $31 \%$ de las personas encuestadas que se encuentran empleados a tiempo completo, mientras que el $18 \%$ son pensionistas o jubilados y el $24 \%$ hacen tareas del hogar. La tasa de paro que se presenta es del $17 \%$, siendo una tasa ligeramente superior a la que se muestra en el primer trimestre del 2017 en Cataluña (15,3\%) (Expansión, 2017).

\subsection{Confort térmico}

Por el estado de degradación que presentaban los edificios dentro del polígono a intervenir, era necesaria la rehabilitación principalmente en las envolventes y las cubiertas de los mismos, con la intención de mejorar energética y térmicamente las viviendas contenidas.

En la mayoría de los edificios, cuando no suponía un sobrecoste excesivamente elevado para los ocupantes de los edificios, se optó por instalar el sistema SATE de recubrimiento con aislamiento en fachada.

Citación: BARÓN, A. et al. Estudio de la percepción del confort térmico, satisfacción residencial y modelo de gestión, de los residentes del área de conservación y rehabilitación energética en la calle Pirineos, Santa Coloma de Gramenet, España. En: Libro de proceedings, CTV 2018. XII Congreso Internacional Ciudad y Territorio Virtual. "Ciudades y Territorios Inteligentes". UNCuyo, Mendoza, 5-7 septiembre 2018. Barcelona: CPSV, 2018, p. 303-318. 
Como resultado, se obtuvo que el $75 \%$ de las personas encuestadas coincidieron en que se sentían exactamente igual con la temperatura de su vivienda una vez realizada la intervención. No obstante, el $22 \%$ declararon sentirse más incómodos con la temperatura de la vivienda después de la intervención. En este sentido, cabe apuntar que algunas personas confesaron sentir un incremento de la temperatura de la vivienda y manifestaron el temor de padecer este aumento de temperatura también en verano. Por lo tanto, estaban expresando su opinión sobre un futuro, el cual desconocen, y no estaban valorando el aumento de temperatura en los meses de invierno y principios de primavera.

Por el contrario, cuando se les preguntaba específicamente por el cambio que habían notado con la temperatura de la vivienda en los meses de invierno, el $22 \%$ respondió haber notado una mejoría en la temperatura. Dentro de los resultados también se pudo observar que aumentaron las temperaturas de las viviendas más frías y que en ningún caso varió la opinión de los encuestados que sentían la temperatura de sus viviendas agradables o moderadamente cálidas. Es decir, se mejoró el confort térmico interior en las viviendas que más lo necesitaban y se mantuvo igual en los casos en los que ya disponían de temperaturas agradables en los meses más fríos.

De las posibles medidas para combatir el frio, las más utilizadas por las personas encuestadas son abrigarse más y encender la calefacción, y en menor medida, el uso de mantas y tomar bebidas calientes. El $17 \%$ de las personas encuestadas contestó que después de la intervención realiza con menos frecuencia estas acciones.

Además, el $62 \%$ de las personas encuestadas afirman sentir las paredes menos frías que antes de la rehabilitación, por lo que se deduce que se está produciendo un menor intercambio de energía interior-exterior.

En cuanto a las medidas activas para mantener el confort térmico interior de las viviendas, el $32 \%$ de las personas encuestadas encendían la calefacción en los meses de invierno antes de la intervención. El porcentaje sobre la utilización de la calefacción después de la rehabilitación bajó un $4 \%$. Además, el $13 \%$ de los encuestados confiesa haberla encendido menos horas que antes de la rehabilitación.

Asimismo, de los que respondieron sobre la cantidad de ingresos que destinaban a pagar las facturas energéticas, el $15 \%$ de las viviendas que viven bajo el umbral de pobreza energética, es decir, que destinan más del $10 \%$ de sus ingresos a pagar las facturas de suministro de energía, notaron una mejoría.

Profundizando aún más en estos datos, resulta que los únicos edificios donde los habitantes han notado mejoría en las condiciones térmicas de sus viviendas corresponden a los edificios de la calle Pirineos ํo 83, o 85 y ํo 87. La orientación de las viviendas que contienen estos edificios tampoco se advierte como una explicación razonable a los resultados encontrados, pues se han producido en viviendas orientadas tanto a norte como a sur. Las únicas semejanzas encontradas entre estos pisos, son el tamaño y el porcentaje de mejora en eficiencia energética que han alcanzado gracias a introducir el correspondiente aislamiento térmico en sus envolventes.

Por un lado, estos pisos comparten la similitud de ser de los más pequeños sobre los que se ha actuado y los que presentan una relación entre los metros lineales de fachada y los metros cuadrados construidos más alta, concretamente, entre el 0,13 y el 0,15 de $\mathrm{ml}$ de fachada por

Citación: BARÓN, A. et al. Estudio de la percepción del confort térmico, satisfacción residencial y modelo de gestión, de los residentes del área de conservación y rehabilitación energética en la calle Pirineos, Santa Coloma de Gramenet, España. En: Libro de proceedings, CTV 2018. XII Congreso Internacional Ciudad y Territorio Virtual. "Ciudades y Territorios Inteligentes". UNCuyo, Mendoza, 5-7 septiembre 2018. Barcelona: CPSV, 2018, p. 303-318. 
m2 de vivienda. Es decir, proporcionalmente estos pisos son más influenciables por las mejoras acaecidos en las envolventes que el resto.

Por otro lado, los edificios en que se ha notado una mejora de temperatura, coinciden con los edificios que más porcentaje de mejora en eficiencia energética han obtenido. Los 3 edificios comparten una mejora superior al $40 \%$ respecto a su situación anterior a la rehabilitación ( $i$ Error! No se encuentra el origen de la referencia.).

Estos casos no necesariamente guardan relación con una mejora en el sistema de calificación energético español, puesto que otros han mejorado su calificación (letra) pero no han notado ninguna mejora. Simplemente los que han notado alguna mejora en la temperatura, son los que han reducido en mayor proporción la demanda de energía necesaria para alcanzar condiciones de confort interior y que por lo tanto, han advertido unos supuestos ahorros en las emisiones de $\mathrm{CO}_{2}$.

Figura 2. Calificación energética y tanto por ciento de mejora por edificio.

\begin{tabular}{|c|c|c|c|c|c|}
\hline & \multicolumn{4}{|c|}{ Calificación energética } & \multirow[b]{2}{*}{$\%$ de Mejora } \\
\hline Dirección edificios & Anterior & $\mathrm{KgCO} 2 / \mathrm{m} 2$ año & Posterior & $\mathrm{KgCO} 2 / \mathrm{m} 2$ año & \\
\hline Pirineus, 83 & $E$ & 34 & D & 19,92 & $41,41 \%$ \\
\hline Pirineus, $85-87$ & $\mathrm{E}$ & 38,72 & $\mathrm{D}$ & 20,17 & $47,91 \%$ \\
\hline Ptge. St Pasqual, 20 & $\mathrm{~F}$ & 42 & $\mathrm{E}$ & 27,71 & $34,02 \%$ \\
\hline Ptge. Pirineus, 8 & $E$ & 33,8 & $E$ & 29,96 & $11,36 \%$ \\
\hline Ptge. Pirineus, 6 & $E$ & 35,38 & $E$ & 32,72 & $7,52 \%$ \\
\hline Ptge. Pirineus, 10 & $\mathrm{~F}$ & 42 & $\mathrm{~F}$ & 42 & $0,00 \%$ \\
\hline Ptge. St Pasqual, 22 & G & 58 & $\mathrm{~F}$ & 43,58 & $24,86 \%$ \\
\hline
\end{tabular}

Fuente: Elaboración propia

El porcentaje de mejora no va a traducirse literalmente en una disminución de las emisiones de $\mathrm{CO}_{2}$ ni en un ahorro de energía, debido a los elevados casos que no cuentan con calefacción o simplemente que no hacen uso de estos sistemas activos porque no pueden permitírselo económicamente. Sin embargo, aparece un tema, no menos importante, que puede vislumbrarse en los edificios donde más se ha mejorado la eficiencia energética, este aspecto ha repercutido directamente en la temperatura de la vivienda y por ende, en una mejora de la confortabilidad térmica de la vivienda y de la calidad de vida de sus habitantes.

\subsection{Satisfacción residencial}

Las encuestas fueron diseñadas para la obtención de datos sobre la satisfacción residencial en tres diferentes escalas: vivienda, edificio y barrio.

El $44.8 \%$ de los encuestados creen que la intervención realizada en las envolventes y cubiertas de su edificio, ayudó poco a mejorar su vivienda, a pesar de este dato, aproximadamente el $30 \%$ de los propietarios que respondieron, creen que su vivienda ha aumentado de valor.

Se obtuvo igual proporción (43\%) en la respuesta sobre dos rangos de incremento del valor de la vivienda: (I) menos de 4.000 euros y (II) entre 4.000 y 9.000 euros. Considerando el costo proporcionado por cada propietario para solventar los gastos de la rehabilitación, lo esperado sería que el incremento de valor pretendido por las personas encuestadas pudiera estar dentro del segundo rango mencionado. Los resultados también muestran la coherencia entre la percepción de las personas encuestadas referente a la valorización de su vivienda y la mejoría

Citación: BARÓN, A. et al. Estudio de la percepción del confort térmico, satisfacción residencial y modelo de gestión, de los residentes del área de conservación y rehabilitación energética en la calle Pirineos, Santa Coloma de Gramenet, España. En: Libro de proceedings, CTV 2018. XII Congreso Internacional Ciudad y Territorio Virtual. "Ciudades y Territorios Inteligentes". UNCuyo, Mendoza, 5-7 septiembre 2018. Barcelona: CPSV, 2018, p. 303-318. 
de la misma, por lo tanto los que contestaron que mejoro Poco su vivienda, también creen que no se valorizó.

Con respecto a la percepción de los encuestados sobre su edificio, resalta que solo el $9 \%$ de ellos cree que su bloque estaba en buen estado de conservación y después de la intervención, el $55 \%$ mencionaron que ha mejorado el estado físico de su edificio.

Se tenía la hipótesis que el proceso de gestión (reuniones e intercambio de ideas respecto a un tema en común y dinámicas barriales) podría generar una mejor relación y cohesión social entre los vecinos directos (edificio) y los del barrio en general. Dicha suposición no presenta los resultados esperados, ya que tan solo el $10 \%$ de las personas encuestadas, menciona que ha mejorado su relación con los vecinos del edificio y el $16 \%$ con los del barrio. Del mismo modo, la intervención no ha logrado, por el momento, mejorar las relaciones de los habitantes españoles con sus vecinos, independientemente de su nacionalidad. Por el contrario, una pequeña parte de los extranjeros sí creen haber reforzado los lazos con sus vecinos.

La mayoría (55\%) de las personas encuestadas llevan más de 10 años viviendo en el barrio de Fondo. Si consideramos que el $42 \%$ opinó que el barrio era regular y el $35 \%$ que era poco adecuado para vivir antes de la rehabilitación, se contextualiza el objetivo plasmado por la Alcaldesa de Santa Coloma de Gramenet sobre la necesidad de dignificar el barrio (Parlón i Gil, 2017). Después de la rehabilitación el $45 \%$ de las personas encuestadas consideran que ha mejorado el barrio, y tan solo el $9 \%$ menciona que la imagen del conjunto residencial ha empeorado. Este es un dato positivo, aunque consideramos que la influencia sobre los habitantes del barrio debió de haber tenido una presencia más integral y no tan solo en el entorno físico (imagen del barrio), ya que el $84 \%$ de las personas encuestadas menciona que su relación con las personas del barrio no ha cambiado. Sin embargo, las personas que perciben una mejor relación con los vecinos del barrio en su mayoría son extranjeros.

Para las siguientes intervenciones (rehabilitaciones de este tipo), se debería reflexionar y buscar estrategias que puedan apropiar e identificar más a los usuarios (beneficiarios) con el proyecto, como también construir vínculos sociales entre ellos. Siendo así que se podría generar una dignificación más integral del barrio y la consolidación de un entorno cohesionado.

\subsection{Gestión del proyecto}

La gestión del proyecto por parte del Ayuntamiento de Santa Coloma de Gramenet es pionera y única en su tipo, ya que rehabilitaciones público-privadas dentro de propiedades privadas no se habían realizado anteriormente en Cataluña. Por lo cual, las respuestas obtenidas en las encuestas realizadas nos ofrecerán una tendencia, que en un futuro podrán servir como semilla para evaluar con mayor profundidad la gestión y política pública implementada dentro del polígono de intervención.

La gestión del proyecto se evaluó sobre dos temáticas particulares: (a) la gestión del Ayuntamiento durante el desarrollo del proyecto, en cuanto a información del proceso, resolución de inquietudes o preguntas de los beneficiarios/usuarios del proyecto, seguimiento de obras y el sistema de pago propuesto y (b) el proyecto de rehabilitación en cuanto a las soluciones propuestas, el resultado y la actuación del personal de obra. Para esta evaluación se utilizó una calificación cualitativa, asociada a una cuantitativa así: Pésimo (2), Malo (3), Bueno (4) y Excelente (5). Dichos valores se utilizan para generar una calificación de los aspectos de gestión evaluados en la encuesta.

Citación: BARÓN, A. et al. Estudio de la percepción del confort térmico, satisfacción residencial y modelo de gestión, de los residentes del área de conservación y rehabilitación energética en la calle Pirineos, Santa Coloma de Gramenet, España. En: Libro de proceedings, CTV 2018. XII Congreso Internacional Ciudad y Territorio Virtual. "Ciudades y Territorios Inteligentes". UNCuyo, Mendoza, 5-7 septiembre 2018. Barcelona: CPSV, 2018, p. 303-318. 
Sobre las estrategias de comunicación para lograr informar a los vecinos sobre el proyecto, el $48.4 \%$ de los encuestados consideraron que la información proporcionada por el Ayuntamiento fue buena, siendo la calificación promedio obtenida para la gestión del Ayuntamiento sobre "información del proceso" de 3.42.

En relación con la resolución de inquietudes, el $29 \%$ de las personas encuestadas consideraron que, por parte del personal técnico del Ayuntamiento, fue mala, sin embargo, el mismo porcentaje de personas contestó a su vez, que la resolución de inquietudes fue buena. En términos generales, la calificación promedio obtenida para la gestión del Ayuntamiento sobre la "resolución de inquietudes" es de 3.10.

Respecto a la supervisión de la obra, el $48 \%$ de los encuestados creen que hubo un buen seguimiento por parte del equipo del Ayuntamiento, obteniendo una calificación general promedio de 3.42 en el "seguimiento de obras".

El sistema de financiamiento propuesto por parte del Ayuntamiento, el cual funge como entidad crediticia, es una de las iniciativas más innovadoras y que sin lugar a duda coadyuvó en gran medida a que se incentivara el proyecto y se consiguiera ejecutar. El $61 \%$ de los vecinos encuestados encuentran el sistema de pago acertado, calificándolo dentro de la categoría bueno, obteniendo una calificación promedio de 3.89. Sin embargo, el 74\% respondió que el coste de la intervención fue desproporcionado, esto tiene sentido tomando los rangos de ingreso mensual familiar mencionados en el apartado anterior y considerando la vulnerabilidad de la población diagnosticada dentro de los estudios previos al proyecto.

En relación con las "soluciones propuestas" el $54 \%$ de las personas encuestadas las califica como buenas, siendo la calificación media de este aspecto igual a 3.33. Con respecto a la evaluación del proyecto, el $50 \%$ cree que hubo un buen resultado, aunque el $38 \%$ opina lo contrario, obteniendo un $22 \%$ con respuestas de malo y $16 \%$ como pésimo. Por lo tanto, frente al "Resultado" la calificación promedio de los encuestados es de 3.47. No obstante, si se diferencia por régimen de tenencia la calificación media de los "resultados" es mayor para quienes están en alquiler (4.0), en relación con los propietarios $(3,3)$. Asimismo los nacidos en el otro país califican el resultado con una media de 3.9, mientras que para los nacidos en España califican el resultado con 3.2 en promedio.

Finalmente, el 55\% de las personas encuestadas consideraron como buena la "actuación del personal de obra" que recibió de calificación media un 3.52.

Lo anterior habla bien del seguimiento, control, limpieza y buen trato por parte de los supervisores y contratistas involucrados en la ejecución de obra, ya que siendo una intervención a gran escala que involucraba mucha convivencia dentro y fuera de los edificios, generalmente ocasiona relaciones ríspidas entre el personal de obra y los propietarios.

Frente a las "expectativas" que generó el proyecto en los vecinos, el $52 \%$ de las personas encuestadas contestaron que están insatisfechas en relación con las expectativas que tenían antes del inicio de las obras y tan solo el $17 \%$ respondió que había superado sus expectativas. Esta insatisfacción en relación al resultado del proyecto se vio reflejada principalmente en los encuestados que son propietarios, lo cual, según algunos comentarios recogidos en campo, se puede deber a diversos factores como: el costo desproporcionado; las molestias ocasionadas durante la obra; la selección de colores de pintura en las fachadas; y/o a la poca sensación de cambio térmico percibido dentro de sus viviendas. El promedio de las "Expectativas" frente al proyecto fue de 1.66 siendo (3) "superó mis expectativas", (2) "Era lo que esperaba" y (1) "Es

Citación: BARÓN, A. et al. Estudio de la percepción del confort térmico, satisfacción residencial y modelo de gestión, de los residentes del área de conservación y rehabilitación energética en la calle Pirineos, Santa Coloma de Gramenet, España. En: Libro de proceedings, CTV 2018. XII Congreso Internacional Ciudad y Territorio Virtual. "Ciudades y Territorios Inteligentes". UNCuyo, Mendoza, 5-7 septiembre 2018. Barcelona: CPSV, 2018, p. 303-318. 
insatisfactorio". Sin embargo, si se tiene en cuenta el régimen de tenencia, la calificación promedio de los propietarios es ligeramente superior (1.67) y si se tiene en cuenta el lugar de nacimiento del encuestado, para los nacidos fuera de España, sus expectativas fueron superadas en mayor medida, con una calificación media de 1.82, frente a una media de 1.56 de los españoles, situación que también fue percibida durante la ejecución de las encuestas.

\section{Conclusiones finales}

Del análisis de las encuestas realizadas a los residentes de los edificios que finalizaron antes de noviembre de 2016 del proyecto de Rehabilitación de los edificios incluidos en el ACR Pirineos, ejecutado por el Ayuntamiento de Santa Coloma de Gramenet, se puede concluir que uno de los mayores logros ha sido el sistema de pago del proyecto, obteniendo una calificación promedio entre los propietarios de 3.89 sobre 5, siendo este el aspecto mejor evaluado, no obstante, teniendo en cuenta los rangos de ingreso mensual familiar y considerando la vulnerabilidad de la población del sector, el $74 \%$ de las personas encuestadas considera que el coste de la intervención fue desproporcionado, encontrándolo alto con respecto al beneficio reportado. Si bien el esquema es bien aceptado por los vecinos, una de las recomendaciones para futuros proyectos de similar envergadura, es encontrar mecanismos de subvención de algún porcentaje de las intervenciones realizadas, así como un seguimiento oportuno de los pagos, de manera que no se acumulen por varios meses, afectando directamente los usuarios.

Figura 3. Vista de la intervención terminada

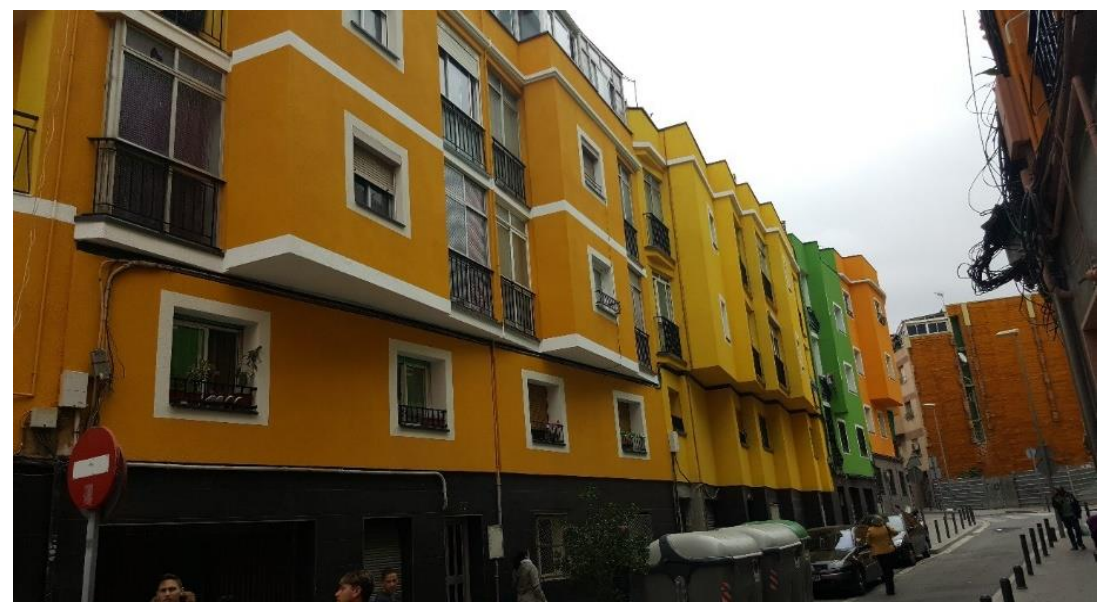

Fuente: Fotografía propia de los autores

La gestión del Ayuntamiento en términos generales se percibe como aceptable, con calificaciones promedio cercanas a 3.4 sobre 5, dejando en evidencia cosas por mejorar, particularmente a nivel del lenguaje de comunicación empleado con los vecinos, que evite generar expectativas diferentes a las planeadas para el proyecto, con alcances puntuales, claros y fáciles de entender para aquellos que desconocen los términos técnicos.

En relación con la satisfacción residencial, dado que el proyecto se encuentra en ejecución, aún no se percibe ningún cambio social en la relación de las personas con sus vecinos, el edificio y el barrio. Aunque la mayoría opina que la imagen del barrio ha mejorado (

Figura 3), consideran que el valor de su vivienda no se ha incrementado. Sería interesante poder analizar después de un periodo de tiempo las incidencias reportadas a la guardia urbana con relación a los delitos contra el orden público, convivencia vecinal, índices de criminalidad y

Citación: BARÓN, A. et al. Estudio de la percepción del confort térmico, satisfacción residencial y modelo de gestión, de los residentes del área de conservación y rehabilitación energética en la calle Pirineos, Santa Coloma de Gramenet, España. En: Libro de proceedings, CTV 2018. XII Congreso Internacional Ciudad y Territorio Virtual. "Ciudades y Territorios Inteligentes". UNCuyo, Mendoza, 5-7 septiembre 2018. Barcelona: CPSV, 2018, p. 303-318. 
denuncias que hayan existido en el área rehabilitada. También sería necesario investigar si se generaron nuevas redes y dinámicas sociales positivas en el barrio y así obtener un diagnóstico y conocer si la "dignificación" (objetivo político) fue real o solo fue un cambio físico y socialmente no logró el impacto esperado.

Respecto al confort térmico general de la vivienda, la mayoría de las personas sienten una temperatura exactamente igual que antes de la rehabilitación. A pesar de ello, hay indicios sobre respuestas más concretas que sugieren una ligera mejoría entre el confort térmico de las viviendas del anterior invierno al posterior a la intervención. Estos datos resultan aún más positivos si consideramos que el último invierno (2016-2017) fue menos cálido que el anterior (2015-2016). (IDESCAT, s.f.) No obstante, debería evaluarse esta tendencia con el paso del tiempo en las distintas épocas del año y controlando los diferentes factores que afectan la percepción del confort térmico.

Las respuestas obtenidas en las encuestas realizadas nos ofrecen una tendencia frente a la percepción de los usuarios, que en un futuro podrán servir para profundizar la evaluación de la gestión y política pública desde una perspectiva de los usuarios y el impacto social generado dentro del polígono de intervención.

Para futuros proyectos de características similares, sería bueno tener en cuenta adicionalmente mediciones de datos objetivos sobre el confort térmico interior de las viviendas y sus consumos energéticos, ya que éstos permitirían evaluar y justificar de una manera más completa el coste de las mejoras acaecidas en este tipo de áreas y edificios degradados.

\section{Bibliografía}

AGENCIA ESTATAL BOLETÍN OFICIAL DEL ESTADO. Boletín Oficial del Estado. (26 de 07 de 2017). Disponible en: https://www.boe.es/diario boe/txt.php?id=BOE-A-2013-6938

AGENCIA ESTATAL BOLETÍN OFICIAL DEL ESTADO. Boletín Oficial del Estado. (26 de 07 de 2017). Disponible en: https://www.boe.es/buscar/act.php?id=BOE-A-2008-10792

AGENCIA ESTATAL BOLETÍN OFICIAL DEL ESTADO. Boletín Oficial del Estado. (26 de 07 de 2017). Disponible en: https://www.boe.es/buscar/doc.php?id=BOE-A-2010-13883

AGENCIA ESTATAL BOLETÍN OFICIAL DEL ESTADO. Boletín Oficial del Estado. (26 de 07 de 2017). Disponible en: https://www.boe.es/buscar/act.php?id=BOE-A-1999-21567

AGENCIA ESTATAL BOLETÍN OFICIAL DEL ESTADO. BOE-A-2008-3657. (26 de 07 de 2017). Disponible en: https://www.boe.es/diario boe/txt.php?id=BOE-A-2008-3657

ASHRAE. Thermal Environmental Conditions for Human Occupancy. (2010).

AYUNTAMIENTO DE SANTA COLOMA DE GRAMENET. Área de Conservación y Rehabilitacion de la Calle Pirineos. Ayuntamiento de Santa Coloma de Gramenet, Área de Urbanismo, Servicios Municipales, Espacio Público, Civismo y Sostenibilidad. 2014.

BANCO DE BUENAS PRÁCTICAS. Renovem els Barris Buena práctica municipal. [en línea] Mayo de 2016. Fecha de consulta: 14 de Marzo de 2017. Disponible en: http://www.gramenet.cat/es/temas/territorio/renovem-els-barris/buena-practica-municipal/

Citación: BARÓN, A. et al. Estudio de la percepción del confort térmico, satisfacción residencial y modelo de gestión, de los residentes del área de conservación y rehabilitación energética en la calle Pirineos, Santa Coloma de Gramenet, España. En: Libro de proceedings, CTV 2018. XII Congreso Internacional Ciudad y Territorio Virtual. "Ciudades y Territorios Inteligentes". UNCuyo, Mendoza, 5-7 septiembre 2018. Barcelona: CPSV, 2018, p. 303-318. 
BARCELONA REGIONAL. Tipología de edificios de viviendas en Santa Coloma. 2012.

BRAGER, G. S., \& DEAR, R. J. (1997). Thermal adaptation in the built environment: a literature review. En: Energy and Buildings, 83-86.

CASTEJÓN, E. NTP 74: Confort térmico. 1983.

COMITÉ TÉCNICO AEN/CTN 81. Ergonomía del ambiente térmico. España. 2006.

EXPANSIÓN. Datosmacro.com [en línea] 20 de 06 de 2017. Disponible en: http://www.datosmacro.com/paro-epa/espana-comunidades-autonomas/cataluna

GARCÍA-ALMIRALL, P. Satisfacción Residencial. Encuesta diseñada. Barcelona: Centre de Política de Sòl i Valoracions. 2012.

GRAHAM, Sh. College of one. New York : Viking, 1967.

GRAHAM, Sh. Review of College Comtemporaries. En: Urban Studies, 1975, III, 4: 243-256.

GRAY, C., \& LARSON, E. Administración de Proyectos. Mexico: McGraw Hill. 2009.

IDESCAT. (s.f.). Instituto de estadística de Catalunya. Disponible en: https://www.idescat.cat

INE. INE. 06 de 04 de 2016. Disponible en: http://www.ine.es/prensa/np965.pdf

INDRAGANTIA, M.; OOKAB, R. \& RIJALC, H. B. Thermal confort in offices in India: Behavioral adaptation and the effect of age and gender. En: Energy and Buildings, 103, 284-295. 2015.

MAE-SECIPI. Metodología de Evaluación de la Cooperación Española. Madrid. 2001.

MARCO AGUILAR, I. Parámetros para la rehabilitación energética de las fachadas en Santa Coloma. Trabajo Fin de Master Tecnología y Arquitectura, ETSAB-UPC. 2012.

PARLÓN I GIL, N. La rehabilitación de viviendas a partir de herramientas urbanísticas: la calle pirineos, Santa Coloma de Gramenet. En: C. B. Congreso de Vivienda Social, Entrevistador. 23 de mayo de 2017.

Planells Pérez, A. (2012). Clasificación y análisis tipológico de edificios residenciales construidos entre 1950 y 1975 en Santa Coloma. Trabajo Final de Master en Tecnología y Arquitectura , ETSAB-UPC, Barcelona.

Project Managment Institute. (2008). PMBOK Cuarta edición. Guía de los Fundamentos para la Dirección de Proyectos. Pennsylvania, EE.UU.: Project Management Institute, Inc.

Sapag Chain, N., \& Sapag Chain, R. (2008). Preparación y Evaluación de Proyectos, Quinta edición. Santiago de Chile, Chile: McGraw- Hill Interamericana.

VILCHES, A.; BARRIOS, Á., \& MOLINA, M. Retrofitting of homes for people in fuel poverty: Approach based on household thermal comfort. En: Energy Policy, 100: 283-291. 2017.

VON BREYMANN, H. La satisfacción residencial como herramienta de evaluación de proyectos de rehabilitación urbana. Madrid, Univ. Politécnica de Madrid / Univ. de Costa Rica. 2014.

Citación: BARÓN, A. et al. Estudio de la percepción del confort térmico, satisfacción residencial y modelo de gestión, de los residentes del área de conservación y rehabilitación energética en la calle Pirineos, Santa Coloma de Gramenet, España. En: Libro de proceedings, CTV 2018. XII Congreso Internacional Ciudad y Territorio Virtual. "Ciudades y Territorios Inteligentes". UNCuyo, Mendoza, 5-7 septiembre 2018. Barcelona: CPSV, 2018, p. 303-318. 